\title{
Espessura corneana central e suas correlações com outros dados biométricos oculares em pacientes portadores de glaucoma congênito
}

\author{
Central corneal thickness and its correlations with other ocular biometric data in patients with \\ congenital glaucoma
}

Marcio Henrique Mendes ${ }^{1}$, Lisandro Sakata ${ }^{2}$, Alberto Jorge Betinjane ${ }^{1}$

\section{RESUMO}

Objetivo: Estudar a distribuição da espessura corneana central e suas correlações com outros dados biométricos em pacientes com glaucoma congênito.

Métodos: Pacientes foram divididos em dois grupos, o A composto por portadores de glaucoma congênito, sendo este subdividido em subgrupos: com estrias de Haab (A1) e sem estrias de Haab (A2). O B representou o grupo controle.

Resultados: $O$ grupo A apresentou diâmetro corneano entre 11 e 15,5 mm, com média de $14,13 \mathrm{~mm}$ e desvio padrão de 1,28 , enquanto o grupo $B$ apresentou valores entre 11,5 e $12,5 \mathrm{~mm}$, com média de 12,01 mm com desvio padrão de 0,09 (t=-8,9723 e $\mathrm{p}=1,5083$ em nível 0,05). Os glaucomatosos apresentaram maiores valores médios de diâmetro axial ( $t=-6,46315, p=9,2498$ em nível de significância de 0,05), e menores valores médios ceratométricos em relação aos controles. O subgrupo A1 apresentou espessura corneana central de $539 \pm 46 \mu \mathrm{m}$, o subgrupo A2 apresentou média de $571 \pm 56 \mu \mathrm{m}$ e o grupo $B$ de $559 \pm 28 \mu \mathrm{m}$ ( $\mathrm{t}=0,43746$ e $\mathrm{p}=0,66291$ ). As correlações entre diâmetro corneano e axial foram positivas nos dois grupos. Já entre diâmetro corneano e ceratometria média foram negativas nos dois grupos.

Conclusão: Os glaucomatosos apresentaram maior média de diâmetro axial e menor média ceratométrica em relação aos controles. Não houve diferença estatisticamente significativa da espessura corneana central. O diâmetro corneano se correlacionou positivamente como diâmetro axial e negativamente com a ceratometria média. Não se pode estabelecer correlações entre espessura corneana central e os demais dados biométricos.

Descritores: Córnea/anatomia \& histologia; Topografia da córnea; Catarata/congênito; Biometria

\begin{abstract}
Purpose:Tostudy the distribution of the central corneal thickness and its correlations with other biometric data in patients with congenital glaucoma.

Methods: Patients had been divided into two groups: group "A", composed of patients with congenital glaucoma, being subdivided in two sub-groups: with Haab striae (A1) and without Haab striae (A2), and group"B" that represented the controls.

Results: The group A presented corneal diameter between 11 and $15.5 \mathrm{~mm}$, with mean of $14.13 \mathrm{~mm}$ and standard deviation (SD) of 1.28 , while group B presented values between 11.5 and $12.5 \mathrm{~mm}$, with average of $12.01 \mathrm{~mm}$ SD of 0.09 ( $t=-8.9723$ and $p=1.5083$ in level 0.05 ). Glaucomatous patients presented greater mean values of axial diameter ( $t=-6.46315, p=9.2498$ with level of significance of 0.05 ), and smaller mean keratometry in relation to the controls. The A1 sub-group presented mean central corneal thickness of $539 \pm 46 \mu \mathrm{m}$, the A2 presented $571 \pm 56 \mu \mathrm{m}$, and Group B $559 \pm 28 \mu \mathrm{m}$ $(t=0.43746$ and $p=0.66291)$. The correlation between corneal and axial diameters was positive in both groups. The correlation between corneal diameter and mean keratometric values was negative in both groups.

Conclusions: Patients with congenital glaucoma presented greater mean of axial diameter and smaller mean keratometric values compared to the controls. No statistical significant difference of the central corneal thickness was demonstrated. Corneal and axial diameters were correlated positively. Corneal diameter was correlated negatively with the mean keratometry. It was not possible to establish correlations between the central corneal thickness and other biometric data.
\end{abstract}

Keywords: Cornea/anatomy \& histology; Corneal topography; Cataract/congenital; Biometry

\section{INTRODUÇÃO}

A perda visual em pacientes com glaucoma congênito (GC) ocorre na maioria dos pacientes de maneira precoce, não somente pelo dano do nervo óptico, mas também pelas alterações do bulbo ocular, particularmente as corneanas, como o edema ou então as roturas na membrana de Descemet (estrias de Haab) ${ }^{(1-2)}$.

O diagnóstico do GC é realizado através de uma semiologia bem conduzida, e quando realizado de maneira precoce muitas vezes impede a progressão da doença $a^{(3)}$.

A tonometria é artifício semiológico de extrema importância para o diagnóstico, porém pode revelar valores errôneos devido às condições corneanas nestas crianças ${ }^{(4-6)}$, ou então pela variação relacionada à idade no mesmo paciente, como demonstrado na literatura ${ }^{(7)}$.
Dentre os fatores que levam à interpretação errônea da pressão intraocular (PIO), figura a espessura corneana central como um dos principais. Estudos realizados em adultos, demonstraram correlação positiva entre o aumento da espessura corneana central (ECC) e os valores obtidos com cada tonômetro(8-10).

Já no que diz respeito à curvatura corneana, sabemos que há associação positiva com a $\mathrm{PIO}^{(11)}$, em que o autor chegou à conclusão que a cada 3 dioptrias há aumento de $1 \mathrm{mmHg}$ na PIO.

Dessa maneira, os valores da PIO nestas crianças, podem estar erroneamente aferidos com maior frequência do que em adultos, devido à possibilidade dos valores de espessura central e curvatura corneanas nestes pacientes estarem demasiadamente alterados.

O objetivo principal foi identificar a distribuição da espessura corneal central em pacientes portadores de glaucoma congênito

Submitted for publication: July 27, 2010

Accepted for publication: February 2, 2011

Study carried out at the Hospital das Clínicas, Faculdade de Medicina, Universidade de São Paulo HC-FMUSP - São Paulo (SP), Brasil.

Physician, Hospital das Clínicas, Faculdade de Medicina, Universidade de São Paulo - USP - São Paulo (SP), Brasil.

Physician, Hospital de Clínicas, Faculdade de Medicina, Universidade Federal do Paraná - UFPR -

Curitiba (PR), Brasil.

Funding: No specific financial support was available for this study.

Disclosure of potential conflicts of interest: M.H.Mendes, None; L.Sakata, None; A.J. Betinjane, None.

Correspondence address: Marcio Henrique Mendes, Rua Barata Ribeiro, 380 - Conj. 36 - São Paulo - SP - 01308-000 - Brazil - E-mail: marciohmendes@yahoo.com.br 
acima de 5 anos de idade, estabelecendo suas correlações com outros parâmetros biométricos avaliados (diâmetro e curvatura corneanas, e diâmetro axial do globo ocular).

\section{MÉTODOS}

Para este estudo foram selecionados pacientes acima de cinco anos de idade, regularmente matriculados e acompanhados no ambulatório de glaucoma congênito do Hospital das Clínicas da Faculdade de Medicina da USP (HC-FMUSP). Todos os pacientes (quando adultos), ou seus pais, ou responsáveis legais, assinaram termo de consentimento para participar do estudo.

Os pacientes, examinados no ano de 2004, foram submetidos a uma consulta de rotina do ambulatório de glaucoma congênito, a qual incluiu anamnese direcionada e exame oftalmológico que constou de: determinação da acuidade visual, exame externo, biomicroscopia do segmento anterior, tonometria, gonioscopia, exame de fundo de olho e determinação de dados biométricos oculares ou seja, curvatura corneana (ceratometria), diâmetro axial (ecobiometria), espessura corneana (paquimetria) e diâmetro corneano.

A ceratometria foi realizada em aparelho autorrefrator modelo KR-8900 ${ }^{\mathrm{TM}}$, da marca Topcon Corporation, Tóquio, Japão.

A ecobiometria foi realizada após a instilação de uma gota de colírio anestésico, sendo obtidos cinco valores do diâmetro axial de cada olho. O dispositivo utilizado foi o aparelho UltraScan ${ }^{\mathrm{TM}}$, Alcon Inc., Hunenberg, Suíça.

A paquimetria ultrassônica foi realizada através do aparelho CompuScan P Ultrasonic Pachymeter System model UPC 1000 ${ }^{\mathrm{TM}}$, Karl Storz, Tübinger, Alemanha. Foram realizadas cinco aferições consecutivas em cada olho (e determinada a média) após a instilação de uma gota de colírio anestésico.

A medida do diâmetro corneano foi realizada com o auxílio de compasso oftalmológico. Os pacientes foram acomodados confortavelmente um uma cadeira, encostando a parte posterior da cabeça na parede e foi determinado o diâmetro corneano horizontal.

Foram convidados a participar do grupo controle, os pacientes portadores de estrabismo, que não apresentavam qualquer outra anormalidade ocular, pacientes estes também regularmente matriculados no HC-FMUSP. Foram seguidos os mesmos parâmetros éticos e técnicos neste grupo.

Todos os pacientes portadores de glaucoma congênito que apresentavam qualquer grau de edema corneano ou pressão intraocular acima de $25 \mathrm{mmHg}$ foram excluídos do estudo, com intuito de preservar as características de cada grupo. Essa exclusão diminui a chance de criar viés na comparação, uma vez que o grau de hidratação estromal interfere de maneira extremamente significativa na resistência e espessura corneanas, modificando de sobremaneira a PIO.

Os pacientes foram divididos em 2 grupos: A e B. O grupo A foi composto por pacientes portadores de glaucoma congênito. Este grupo foi subdividido em pacientes com estrias de Haab (subgrupo A1) e sem estrias de Haab (subgrupo A2) com o objetivo de realizar algumas comparações separadamente. O grupo B representou o grupo controle.

A avaliação estatística foi realizada através do programa Windows Excel ${ }^{\circledR}$, Microsoft, Redmond, Washington, EUA.

\section{RESULTADOS}

O grupo de pacientes com glaucoma congênito foi composto por 61 pacientes (grupo A). A idade neste grupo A variou de 5 a 34 anos, com média de 14,5 anos e desvio padrão de 7,93. Já o grupo controle (grupo B) foi representado por 24 pacientes, com idade variando de 5 a 22 anos, com média de idade de 12,08 anos e desvio padrão de 6,6. O teste t de Student não revelou diferença estatisticamente significante entre as médias etárias dos dois grupos.

Houve diferença estatisticamente significativa no diâmetro corneano entre os dois grupos, tendo o grupo A apresentado valores entre 11 e 15,5 mm, com média de 14,13 mm com desvio padrão de
1,28, enquanto o grupo B apresentou valores entre 11,5 e 12,5 mm, com média de 12,01 mm com desvio padrão de 0,09. Teste t de Student revelou $t=-8,9723$ e $p=1,5083$ em nível 0,05.

Quanto à distribuição da espessura corneana central (Gráfico 1), o subgrupo $A 1$, representado por 32 pacientes glaucomatosos com estrias de Haab, apresentou média de $539 \pm 46$ micrômetros (micra), o subgrupo A2, composto por 27 pacientes, apresentou média de $571 \pm 56$ micra e o grupo B de $559 \pm 28$ micra. Aplicando-se o t de Student, não se observou diferença estatisticamente significante entre essas médias.

Também não foi observada diferença estatisticamente significante entre a espessura corneana central dos dois grupos; $t=0,43746$ e $p=0,66291$. Analisando-se o diâmetro axial entre os dois grupos $(A$ e $B$ ), obteve-se diferença estatisticamente significante $t=-6,46315$, $p=9,2498$ em nível de significância de 0,05 , sendo a média dos valores maior no grupo com glaucoma congênito.

Houve diferença estatisticamente significante entre os dois grupos quanto à ceratometria média, sendo esta menor no grupo com glaucoma congênito, sendo de 41,95, desvio de padrão de 2,37, neste grupo. Em relação ao grupo normal, os valores observados foram média de 43,88 e desvio padrão de 1,75 e no grupo controle. $t=3,53077, p=0,00069$.

A regressão linear de Pearson foi utilizada para comparação entre diversos parâmetros dos dois grupos, tais como espessura corneana central, diâmetro corneal e diâmetro axial. As correlações entre diâmetro corneano e diâmetro axial foram positivas em ambos os grupos (Gráfico 2). Já entre diâmetro corneano e ceratometria média foram negativas em ambos os grupos.

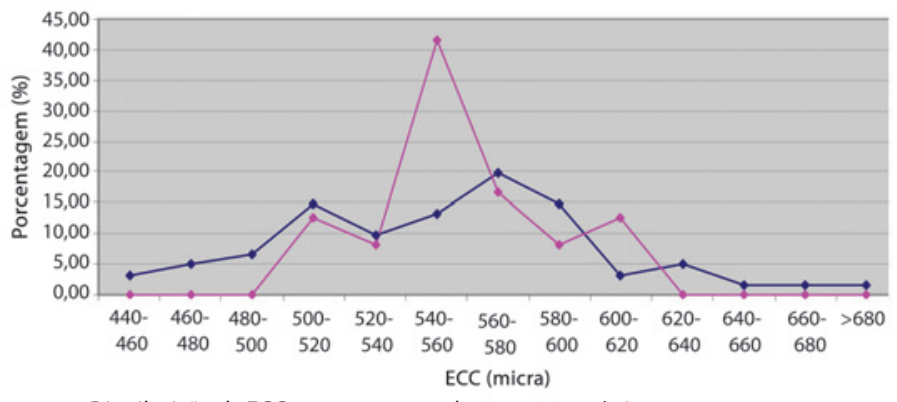

Distribuição da ECC no grupo com glaucoma congênito Distribuição da ECC no grupo controle

Gráfico 1. Distribuição da espessura corneana central (ECC) em pacientes com glaucoma congênito e grupo controle.

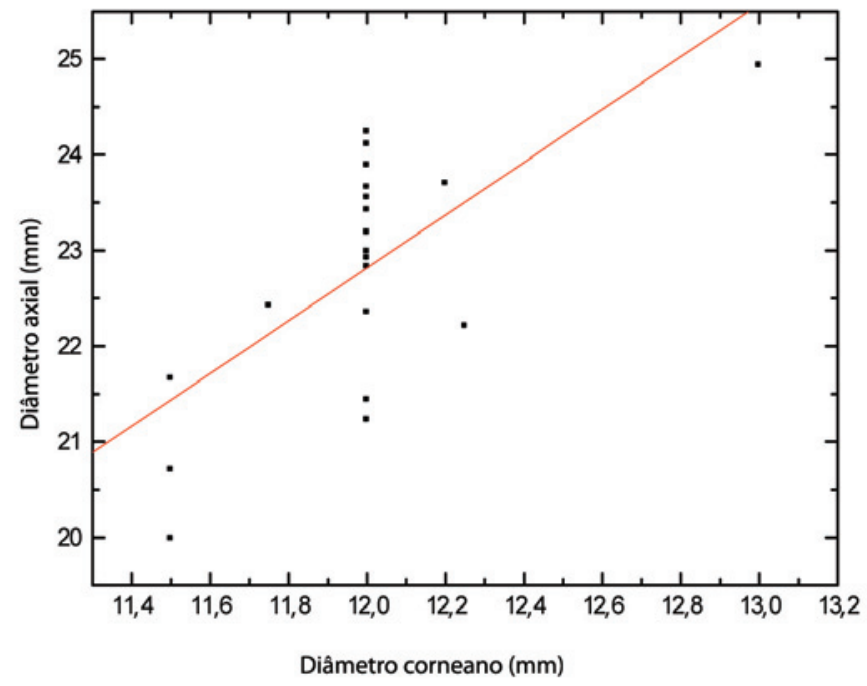

Gráfico 2. Diâmetro corneano x diâmetro axial no grupo controle. 
Tabela 1. Correlações lineares de Pearson quanto aos parâmetros estudados

\begin{tabular}{|c|c|c|}
\hline Correlação & Grupo de glaucoma congênito primário & Grupo controle \\
\hline Diâmetro corneal X diâmetro axial & Positiva $R=0,358 \quad P=0,009$ & Positiva $R=0,687 \quad P=0$ \\
\hline Diâmetro corneal X ceratometria média & Negativa $R=-0,411 P=0,024$ & Negativa $R=-0,445 P=0,032$ \\
\hline Diâmetro axial X espessura corneal central & Não há correlação $R=0 \quad P=0,995$ & Negativa $R=-0,213 \quad P=0,353$ \\
\hline Ceratometria média $X$ espessura corneal central & Positiva $R=0,135 \quad P=0,304$ & Negativa $R=-0,262 \quad P=0,226$ \\
\hline
\end{tabular}

Fonte: Hospital das Clínicas da FMUSP, 2005, São Paulo, Brasil

As demais correlações estudadas foram fracas ou não foram evidenciadas. Houve correlação entre diâmetro axial e ceratometria média no grupo controle, a qual foi nitidamente negativa.

A tabela 1 demonstra todas as correlações estudadas, e os valores das mesmas.

\section{DISCUSSÃO}

No glaucoma congênito, uma propedêutica completa se faz necessário para o diagnóstico e o seguimento da doença. Assim, entre os parâmetros importantes está a PIO. Entretanto, muitas vezes a determinação do valor real da PIO em crianças e jovens portadores de glaucoma congênito é de dificil obtenção levando em conta os métodos de exames comumente usados na tonometria.

A maioria dos autores concorda que os valores tonométricos sofrem influência da ECC, havendo associação positiva entre a ECC e a $\mathrm{PIO}^{(12-14)}$, demonstrada por Wolfs et al. em 1997. Embora haja bem definida esta mudança da PIO em relação a ECC, Feltgen et al. concluíram não haver erro sistemático na medição da PIO na dependência da mudança da ECC (15), não havendo, assim, necessidade de correção da mesma para as diferentes classes de ECC. As exceções à regra são os casos em que há edema corneano, observando-se significativo aumento da ECC e subestimação da PIO devido à diminuição da resistência tecidual corneana relacionada ao acúmulo de líquido no estroma corneano. Daí a importância do estudo dos dados biométricos destes olhos glaucomatosos em relação aos dos olhos normais, constituindo, assim, importantes artifícios no seguimento destes pacientes.

A comparação da espessura corneana média em olhos portadores de glaucoma congênito e olhos normais não revelou diferenças estatisticamente significativas. Nossos resultados são semelhantes aos obtidos por Borges que estudou crianças até 2 anos de idade ${ }^{(16)}$.

Já no que diz respeito ao diâmetro axial e ao diâmetro corneano encontrou-se diferença estatisticamente significatica entre os dois grupos de olhos normais e com glaucoma, sendo maiores os valores médios no grupo de pacientes com glaucoma congênito em comparação com o grupo controle, concordando com os dados obtidos em outros estudos ${ }^{(17-18)}$. No presente estudo, não foi observada correlação entre o diâmetro axial e a espessura corneana central, tendo os mesmos resultados sido observados por outros autores ${ }^{(14,19,20)}$ que também concluíram ser a ECC uma variável independente, não significantemente associada às outras variáveis biométricas oculares.

Como exceção houve correlação entre diâmetro corneano e ECC, sendo demonstrada correlação negativa discreta entre esses dois dados biométricos, havendo concordância com a literatura. Tais dados sugerem possibilidade de que o crescimento corneano, independente do crescimento do bulbo ocular, se correlacione com os menores valores da ECC(16).

\section{CONCLUSÃO}

Neste estudo observou-se que os olhos com glaucoma congênito primário não tiveram espessura corneana média maior que a do grupo controle na amostra estudada. Os olhos glaucomatosos apresentaram maior média de diâmetro axial e menor ceratometria média.
Pode-se afirmar que o presente estudo reforça a necessidade de se levar em conta a biometria ocular no controle evolutivo da doença, uma vez que a determinação da tonometria nem sempre revela valores fidedignos ao estado evolutivo do caso. Apesar da média da espessura corneana central não diferir entre os dois grupos e a mesma não se correlacionar com os demais dados biométricos, ressalta-se a necessidade da sua mensuração, na tentativa de estimarmos de maneira mais aproximada a PIO nestes olhos.

\section{REFERÊNCIAS}

1. Shields MB, Ricth R, Krupin T. Classifications of the glaucomas. In: Ritch R, Shields MB, Krupin T, editors. The glaucomas. $2^{\text {nd }}$ ed. St. Louis: Mosby; 1989. Vol. II:36 p.717-25.

2. Dickens CS, Hoskins HD. Diagnosis and treatment of congenital glaucoma. In: Ritch R, Shields MB, Krupin T, editors. The glaucomas. $2^{\text {nd }}$ ed. St. Louis: Mosby; 1989. Vol. II: 33, p.739-52.

3. Kass MA, Heuer DK, Higginbotham EJ, Johnson CA, Keltner JL, Miller JP, et al. The Ocular Hypertension Treatment Study: a randomized trial determines that topical ocular hypotensive medication delays or prevents the onset of primary open angle glaucoma. Arch Ophthalmol. 2002;120(6):701-13; discussion 829-30. Comment in: Arch Ophthalmol. 2004; 122(7):1088-9; author reply 1089. Arch Ophthalmol. 2003;121(7):1070; author reply 1070.

4. Doughty MJ, Zaman ML. Human corneal thickness and its impact on intraocular pressure measures: a review and meta-analysis approach. Surv Ophthalmol. 2000;44(5):367-408.

5. Wolfs RC, Klaver CC, Vingerling JR, Grobbee DE, Hofman A, de Jong PT. Distribution of central corneal thickness and its association with intraocular pressure: the Rotterdam Study. Am J Ophthalmol. 1997;123(6):767-72.

6. Whitacre MM, Stein RA, Hassanein K. The effect of corneal thickness on applanation tonometry. Am J Ophthalmol. 1993;115(5):592-6.

7. Brandt JD, Beiser JA, Kass MA, Gordon MO. Central corneal thickness in the Ocular Hypertension Treatment Study (OHTS). Ophthalmology. 2001;108(10):1779-88.

8. Tai TY, Mills MD, Beck AD, Joos KM, Ying GS, Liu C, Piltz-Seymour JR. Central corneal thickness and corneal diameter in patients with childhood glaucoma. J Glaucoma. 2006; 15(6):524-8.

9. Martinez-de-la-Casa JM, Garcia-Feijoo J, Vico E, Fernandez-Vidal A, Benitez del Castillo JM, Wasfi M, Garcia-Sanchez J. Effect of corneal thickness on dynamic contour, rebound, and goldmann tonometry.J. Ophthalmology. 2006;113(12):2156-62

10. Martinez-de-la-Casa JM, Garcia-Feijoo J, Fernandez-Vidal A, Mendez-Hernandez C, Garcia-Sanchez J. Ocular response analyzer versus Goldmann applanation tonometry for intraocular pressure measurements. Invest Ophthalmol Vis Sci. 2006;47(10):4410-4

11. Mark HH. Corneal curvature in applanation tonometry. Am J Ophthalmol. 1973;76(2):223-4.

12. Wolfs RC, Klaver CC, Vingerling JR, Grobbee DE, Hofman A, de Jong PT. Distribution of central corneal thickness and its association with intraocular pressure: The Rotterdam Study. Am J Ophthalmol. 1997;123(6):767-72.

13. Whitacre MM, Stein RA, Hassanein K. The effect of corneal thickness on applanation tonometry. Am J Ophthalmol. 1993;115(5):592-6.

14. Alsbirk PH. Primary glaucoma in Greenland (Umanaq district). I. Introduction. The normal intraocular pressure. Acta Ophthalmol (Copenh). 1970;48(6):1061-79.

15. Feltgen N, Leifert D, Funk J. Correlation between central corneal thickness, applanation tonometry, and direct intracameral IOP readings. Br J Ophthalmol. 2001;85(1):85-7.

16. Borges MJH. Determinação da espessura central da córnea, com paquímetro ultrasônico, e sua relação com os diâmetros axial e corneano em olhos de crianças portadoras de glaucoma congênito [tese]. São Paulo: Faculdade de Medicina da Universidade de São Paulo; 2002

17. Carvalho CA, Calixto N. Semiologia do glaucoma congênito. In: Comissão dos anais do Congresso (Eds.). Anais do XV Congresso Brasileiro de Oftalmologia. São Leopoldo: Oficinas gráficas Rotermund;1969. p.105-74.

18. Betinjane AJ, Carvalho CA. Significado do diâmetro corneano e do diâmetro axial total do globo ocular no controle evolutivo do glaucoma congênito. Rev Bras Oftalmol.1994; 53(1):35-9.

19. Ehlers N, Bramsen T, Sperling S. Applanation tonometry and central corneal thickness. Acta Ophthalmol (Copenh), 1975:53(1):34-43.

20. Mello PR; Meirelles SH; Moraes Júnior HV. [Correlation between central corneal thickness and axial length in patients with glaucoma an normal eyes]. Arq Bras Oftalmol. 2009;72(4): 497-502. Portuguese 